\title{
Extracellular Matrix Remodeling in Vascular Development and Disease
}

\author{
Kyoko Imanaka-Yoshida
}

\begin{abstract}
Blood vessels constantly subjected to mechanical stress have well-developed elastic fiber-rich frameworks, which contribute to the elasticity and distensibility of the vascular wall. Destruction of the fibrous structure due to genetic predisposition as well as acquired disorders such as Kawasaki disease often induces irreversible dilation of blood vessels, e.g., aneurysm formation. In addition to their structural role, extracellular matrix molecules also provide important biological signaling, which influences various cellular functions. Among them, increased attention has been focused on matricellular proteins, a group of nonstructural extracellular matrix (ECM) proteins highly upregulated in active tissue remodeling, serving as biological mediators by interacting directly with cells or regulating the activities of growth factors, cytokines, proteases, and other ECM molecules. Tenascin-C (TNC) is a typical matricellular protein expressed during embryonic development and tissue repair/regeneration in a spatiotemporally restricted manner. Various growth factors, pro-inflammatory cytokines, and mechanical stress upregulate its expression. TNC controls cell adhesion, migration, differentiation, and synthesis of ECM molecules. Our recent results suggest that TNC may not only play a significant role in the recruitment of smooth muscle/ mural cells during vascular development, but also regulate the inflammatory response during pathological remodeling. TNC may be a key molecule during vascular development, adaptation, and pathological tissue remodeling.
\end{abstract}

\section{Keywords}

Tenascin • Extracellular matrix $\bullet$ Coronary artery $\bullet$ Aorta

K. Imanaka-Yoshida $(\bowtie)$

Department of Pathology and Matrix Biology, Mie University Graduate School of Medicine, Tsu, Mie 514-8507, Japan

Mie University Research Center for Matrix Biology, Tsu, Mie 514-8507, Japan

e-mail: imanaka@doc.medic.mie-u.ac.jp

T. Nakanishi et al. (eds.), Etiology and Morphogenesis of Congenital Heart Disease,

DOI 10.1007/978-4-431-54628-3_29 


\subsection{Introduction}

Tissue, including the cardiovascular system, is composed of diverse cells and the extracellular matrix (ECM) synthesized by those cells. Several ECM molecules form a fibrous framework and provide structural support for the tissue. Blood vessels constantly subjected to mechanical stress have a well-developed fibrous framework, which contributes to the elasticity and distensibility of the vascular wall in concert with vascular smooth muscle cells. Highly ordered structures consisting of cells and fibrous elements are formed during development and are remodeled during tissue repair/regeneration after injury. In addition to their physical role, several ECM molecules provide important biological signaling, which influences various cellular functions in physiological and pathological tissue remodeling. In particular, ECM, termed matricellular protein, has attracted increasing attention as a biological mediator. Tenascin-C (TNC) is a prototype matricellular protein expressed during embryonic development and tissue repair after injury. This chapter will focus on the role of TNC in vascular development, especially coronary arteries and the aorta.

\subsection{Extracellular Matrix in Vascular Wall}

Blood vessels have abundant fibrous matrix tissue: well-developed elastic fibers in the medial layer and rich collagen fibers in adventitia. It is known that several gene mutations related to these fibrous components cause vascular fragility, eventually leading to aneurysm formation or dissection. For example, the collagen gene and fibrillin-1 gene, which is important for microfibril formation, have been identified as the genes responsible for Ehlers-Danlos syndrome (reviewed in [1]) and Marfan's syndrome [2], respectively. In addition to genetic predisposition, inflammation of blood vessels in acquired disease may induce fragmentation and destruction of normal elastic fibers in the vascular wall and causes irreversible dilation of blood vessels. For example, coronary aneurysm formation is sometimes seen in patients with Kawasaki vasculitis, one of the most common acquired heart diseases in children. Evidently, the structural support by fibrous ECM is essential to maintain the proper morphology and function of blood vessels.

Besides these fibrous elements, unique ECM molecules, matricellular protein [3], have attracted considerable attention. The matricellular proteins have common unique properties: (1) do not contribute directly to structures such as fibrils or basement membranes; (2) high levels of expression during embryonic development and in response to injury; and (3) binding to many cell surface receptors, components of ECM, growth factors, cytokines, and proteases [4]. This is a growing family originally including SPARC, tenascin, and thrombospondin [3]. 


\subsection{Tenascin-C in Vascular System}

Tenascins are a family of four multimeric extracellular matrix glycoproteins: tenascin-C, X, R, and W [5]. The first member, tenascin-C (TNC), is a typical matricellular protein. It is a huge molecule of about $220-400 \mathrm{kDa}$ as an intact monomer and is assembled with a hexamer. The molecule consists of an N-terminal assembly domain, followed by EGF-like repeats, constant and alternatively spliced fibronectin type III repeats, and a C-terminal fibrinogen-like globular domain. Several receptors including integrins, EGFR, annexin II, syndecan-4, and toll-like receptor 4 (TLR-4) bind to the respective domains of TNC and transmit multiple signals (see [6]). Numerous studies have shown that TNC can control the balance of cell adhesion and de-adhesion, cell motility, proliferation, differentiation, and survival (reviewed in [5-7]). Recently, the role of TNC in the modulation of inflammation is highlighted [8].

Tenascin-C is found in many developing organs, including the cardiovascular system, but is often restricted transiently to specific sites, for example, near migrating cells and at sites of epithelial-mesenchymal/mesenchymal-epithelial transition. In normal adults, tenascin-C expression is sparsely detected; however, marked expression is seen in injury, regeneration, and cancer at sites where the tissue structure is being dynamically remodeled. Various factors, including growth factors and pro-inflammatory cytokines, can activate TNC expression (reviewed in [9]). It is particularly of interest that mechanical stress is an important inducer of TNC. Moreover, it is also noteworthy that TNC itself is an elastic molecule and may contribute to tissue elasticity [10].

As well as in other tissue, the expression of TNC in the normal vascular wall is low and upregulated in pathological conditions. The major source is medial smooth muscle cells [11]. However, TNC in the vascular system appears more complex in contrast to the heart [7]. For example, constitutive expression of TNC is observed in the medial layer of the abdominal aorta of normal adult mice but not in the thoracic aorta [12].

\subsubsection{Development of Aorta and Tenascin-C}

The origin of vascular smooth muscle cells (VSMC) of the aorta is heterogeneous [13]. The second heart field gives rise to VSMC of the root of the aorta. The cardiac neural crest contributes ascending and arch portions of the aorta. The origin of VSMC of the descending aorta is more complex. Primitive VSMC of the thoracic aorta originate from the lateral plate mesoderm and are replaced by cells derived from the paraxial mesoderm (somites). Moreover, individual somites build up restricted spatial domains of the "segmental" aortic wall. However, no evident segmental expression pattern of TNC is observed during development of the aorta. In E12-13 mouse embryos, very weak expression of TNC is observed in the ascending aorta and pulmonary truncus. Whereas elastic fibers in the medial layer of the aorta become mature around E12-13, the expression of TNC is upregulated after ED14-15 (Fig. 29.1) when the systemic circulatory system is established. This upregulated expression of TNC may reflect the increased hemodynamic stress on the aortic wall. 


\section{Tenascin-C in developing aorta}

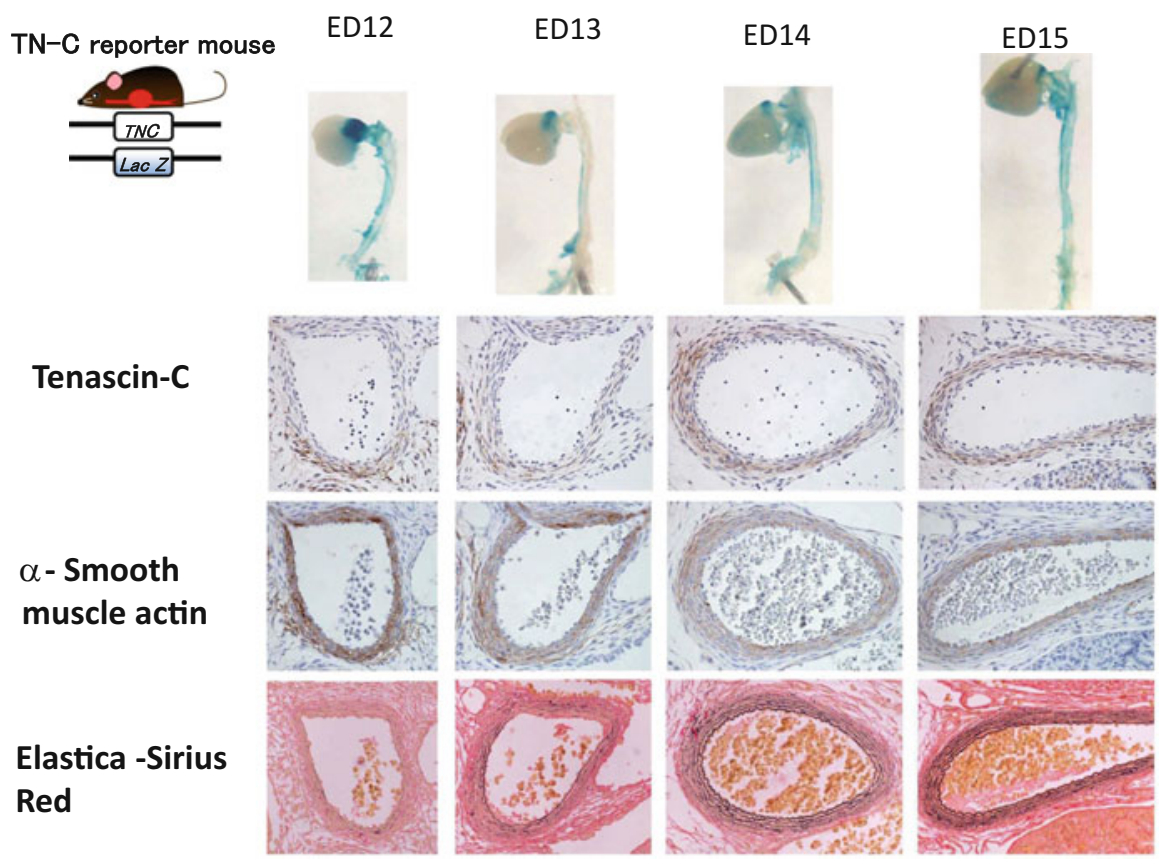

Fig. 29.1 Expression pattern of TNC during development of the aorta. Whole mount lacZ staining and histological sections of descending portion of the thoracic aorta of TNC-reporter mouse embryos at ED12-15. The sections were immunostained with anti-TNC or anti- $\alpha$-smooth muscle actin or stained with elastica sirius red

\subsubsection{Development of Coronary Artery and Tenascin-C}

Coronary vessels are formed with the cells originating mostly from extracardiac tissue known as the proepicardial organ (PE) (see [14] for review). During coronary development, strong expression of TNC is observed, closely associated with thickening of the medial layer when the primitive coronary vasculature connects with the aortic sinuses [15], suggesting a significant role of TNC in maturation of the wall of coronary arteries. Indeed, TNC accelerates the differentiation of mesenchymal cells of PE to smooth muscle cells in culture [15]. Maturation of the vascular wall is regulated by various signaling pathways. In particular, the PDGF-BB/PDGFR- $\beta$ signaling loop is known to be a key regulator of smooth muscle cell recruitment. In vitro, TNC amplifies crosstalk signaling between integrin $\alpha v \beta 3$ and PDGF receptor (PDGFR) $-\beta$ in smooth muscle cells, followed by enhancing cell proliferation and migration [16]. TNC may promote smooth muscle precursor expansion and differentiation in maturation of the vascular wall by enhancing PDGF-BB/PDGFR- $\beta$ signaling (Fig. 29.2). 


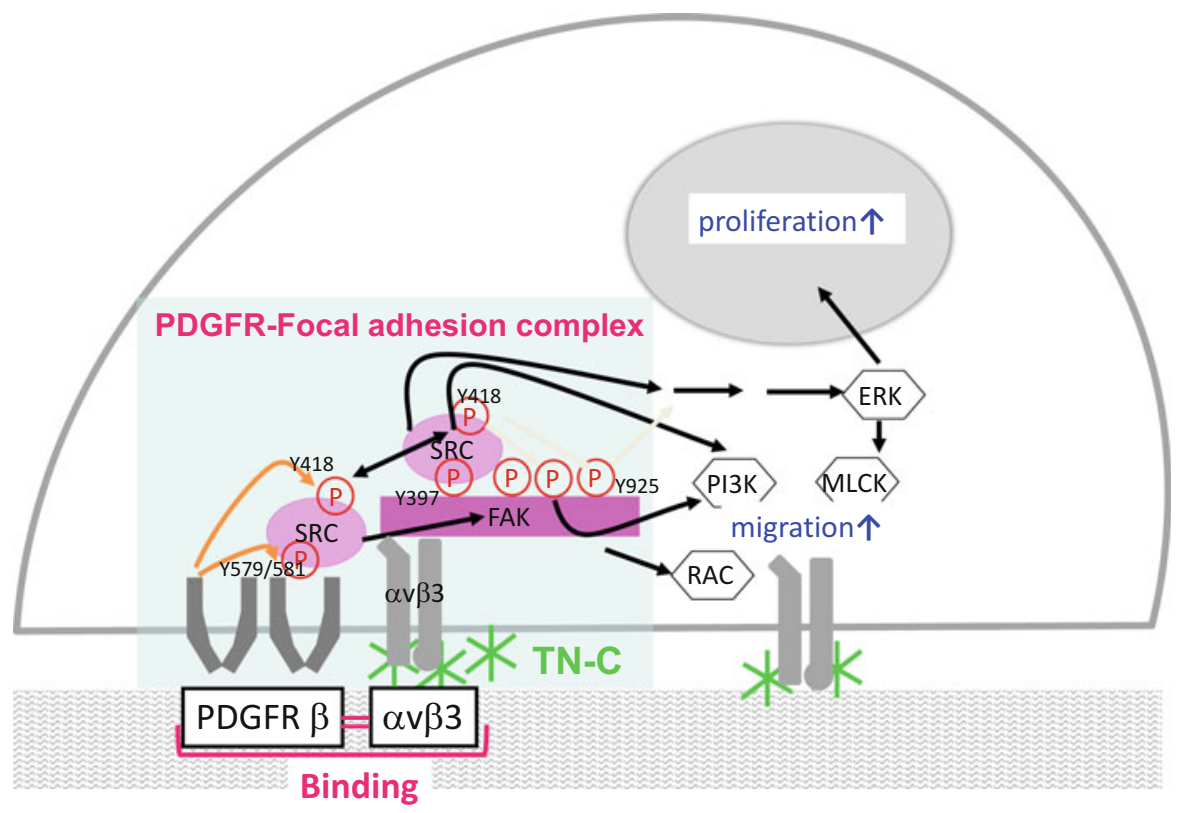

Fig. 29.2 Crosstalk signaling between TNC/integrin $\alpha v \beta 3$ and PDGF-BB/PDGFR- $\beta$ in vascular smooth muscle cells (Adapted from Ref. [7])

\subsection{Future Direction and Clinical Implications}

The characteristic spatiotemporally restricted expression of TNC has suggested its significant role during embryonic development. Several in vitro functional assays support this possibility. Although the grossly normal phenotype of knockout mice suggests the importance of redundancy and compensatory mechanisms during embryonic development, it is not straightforward to understand its molecular function. Meanwhile, TNC expression is linked to a range of vascular diseases, such as aortic aneurysm, acute aortic dissection, and Kawasaki disease (reviewed in $[11,17]$, also see Yoshikane et al. in this proceeding). Increasing numbers of studies have reported that TNC is highly upregulated, associated with inflammation and destruction of the vascular wall, suggesting that TNC may be a diagnostic biomarker. Furthermore, we have succeeded in endovascular treatment of a rat aneurysm model with a TNC-coated coil [18]. Although TNC could contribute to both favorable and undesirable effects during pathological processes in a contextdependent manner, it could be a potential therapeutic target for vascular disease.

Acknowledgments The author thanks M. Hara and M. Namikata for providing technical assistance. This work was supported in part by a research grant for intractable diseases from the Ministry of Health, Labor and Welfare of Japan, and a Grant-in-Aid for Scientific Research from the Ministry of Education, Culture, Sports, Science and Technology of Japan. 
Open Access This chapter is distributed under the terms of the Creative Commons AttributionNoncommercial 2.5 License (http://creativecommons.org/licenses/by-nc/2.5/) which permits any noncommercial use, distribution, and reproduction in any medium, provided the original author(s) and source are credited.

The images or other third party material in this chapter are included in the work's Creative Commons license, unless indicated otherwise in the credit line; if such material is not included in the work's Creative Commons license and the respective action is not permitted by statutory regulation, users will need to obtain permission from the license holder to duplicate, adapt or reproduce the material.

\section{References}

1. Byers PH, Murray ML. Ehlers-Danlos syndrome: a showcase of conditions that lead to understanding matrix biology. Matrix Biol. 2014;33:10-5.

2. Dietz HC, Cutting GR, Pyeritz RE, et al. Marfan syndrome caused by a recurrent de novo missense mutation in the fibrillin gene. Nature. 1991;352:337-9.

3. Sage EH, Bornstein P. Extracellular proteins that modulate cell-matrix interactions. Sparc, tenascin, and thrombospondin. J Biol Chem. 1991;266:14831-4.

4. Bornstein P. Matricellular proteins: an overview. J Cell Commun Signal. 2009;3:163-5.

5. Chiquet-Ehrismann R, Tucker RP. Tenascins and the importance of adhesion modulation. Cold Spring Harb Perspect Biol. 2011;3.

6. Midwood KS, Orend G. The role of tenascin-c in tissue injury and tumorigenesis. J Cell Commun Signal. 2009;3:287-310.

7. Imanaka-Yoshida K. Tenascin-c in cardiovascular tissue remodeling. Circ J. 2012;76:2513-20.

8. Udalova IA, Ruhmann M, Thomson SJ, et al. Expression and immune function of tenascin-c. Crit Rev Immunol. 2011;31:115-45.

9. Tucker RP, Chiquet-Ehrismann R. The regulation of tenascin expression by tissue microenvironments. Biochim Biophys Acta. 2009;1793:888-92.

10. Marin JL, Muniz J, Huerta M, et al. Folding-unfolding of fn-iii domains in tenascin: an elastically coupled two-state system. J Biomech. 2003;36:1733-7.

11. Imanaka-Yoshida K, Yoshida T, Miyagawa-Tomita S. Tenascin-c in development and disease of blood vessels. Anat Rec. 2014;297:1747-57.

12. Kimura T, Furusho A, Ito $\mathrm{S}$ et al. Tenascin $\mathrm{c}$ protects aorta from acute dissection in mice. Sci Rep. 2014;4:4051.

13. Majesky MW. Developmental basis of vascular smooth muscle diversity. Arterioscler Thromb Vasc Biol. 2007;27:1248-58.

14. Nakajima Y, Imanaka-Yoshida K. New insights into the developmental mechanisms of coronary vessels and epicardium. Int Rev Cell Mol Biol. 2013;303:263-317.

15. Ando K, Takahashi M, Yamagishi T, et al. Tenascin c may regulate the recruitment of smooth muscle cells during coronary artery development. Differentiation. 2011;81:299-306.

16. Ishigaki T, Imanaka-Yoshida K, Shimojo N, et al. Tenascin-c enhances crosstalk signaling of integrin alphavbeta3/pdgfr-beta complex by src recruitment promoting pdgf-induced proliferation and migration in smooth muscle cells. J Cell Physiol. 2011;226:2617-24.

17. Gaengel K, Genove G, Armulik A, et al. Endothelial-mural cell signaling in vascular development and angiogenesis. Arterioscler Thromb Vasc Biol. 2009;29:630-8.

18. Toma N, Imanaka-Yoshida K, Takeuchi T, et al. Tenascin-c-coated platinum coils for acceleration of organization of cavities and reduction of lumen size in a rat aneurysm model. $\mathrm{J}$ Neurosurg. 2005;103:681-6. 